\title{
Monomer Abundance Distribution Patterns as a Universal Biosignature: Examples from Terrestrial and Digital Life
}

\author{
Evan D. Dorn ${ }^{1}$, Kenneth H. Nealson ${ }^{2}$, Christoph Adami ${ }^{1,3}$ \\ ${ }^{1}$ Digital Life Laboratory 136-93, California Institute of Technology \\ ${ }^{2}$ Department of Earth Sciences, University of Southern California \\ ${ }^{3}$ Keck Graduate Institute of Applied Life Sciences
}

\begin{abstract}
Keywords: artificial life, amino acids, carboxylic acids, astrobiology, exobiology, evolution, meteorites

Corresponding Author:

Christoph Adami

Keck Graduate Instititute

Claremont, CA 91711

Email: adami@kgi.edu

Phone: (+)909-607-9853
\end{abstract}




\begin{abstract}
Organisms leave a distinctive chemical signature in their environment because they synthesize those molecules that maximize their fitness. As a result, the relative concentrations of related chemical monomers in life-bearing environmental samples reflect, in part, those compounds' adaptive utility. In contrast, rates of molecular synthesis in a lifeless environment are dictated by reaction kinetics and thermodynamics, so concentrations of related monomers in abiotic samples tend to exhibit specific patterns dominated by small, easily formed, low-formation-energy molecules. We contend that this distinction can serve as a universal biosignature: the measurement of chemical concentration ratios that belie formation kinetics or equilibrium thermodynamics indicates the likely presence of life. We explore the features of this biosignature as observed in amino acids and carboxylic acids, using published data from numerous studies of terrestrial sediments, abiotic (spark, UV, and high-energy proton) synthesis experments, and meteorite bodies. We then compare these data to the results of experimental studies of an evolving digital life system. We observe the robust and repeatable evolution of an analogous biosignature in a digital lifeform, suggesting that evolutionary selection necessarily constrains organism composition and that the monomer abundance biosignature phenomenon is universal to evolved biosystems.
\end{abstract}

\title{
Introduction
}

The environmental concentrations of related chemical species carry information about their origin and synthesis. Relative rates of synthesis for individual chemical species differ between biotic and abiotic sources. Where molecules are synthesized by abiotic processes, rates of formation are constrained by the laws of thermodymamics and kinetics, resulting in a distribution of molecules dominated by low molecular weight and kinetically-allowable species. Organisms, on the other hand, contain catalysts (e.g., in terrestrial biota, enzymes) and expend energy to synthesize specifically those molecules they need for survival and competition. In the presence of life, therefore, some specific complex and high-formation-energy molecules are synthesized rapidly because they convey a fitness benefit. If the population is 
large enough, the organisms' chemical composition can become measurable in, or even dominant over, the chemical composition of the environment. In effect, the action of evolution on genomes can imprint a signature on environmental chemistry because fit genomes synthesize molecules at rates that reflect their utility for propagating the genome within the given environment, thus altering the molecules' distribution in the environment. If the population is large enough to contribute to bulk concentration and if these compounds are not quickly destroyed (non-biologically mediated diagenesis), biosynthesis (i.e., biogenesis) may be a significant factor governing the observed concentrations in a sample. Similarly, biologicallymediated diagenesis may affect environmental concentrations in a way that reflects evolved metabolic activity and, therefore, selection.

The distribution of a set of molecules in an environmental sample, therefore, may indicate the presence or absence of life. The principle that patterns of molecular abundance within a family of molecules to distinguish abiotic and biotic sources of those molecules has been discussed previously (Lovelock, 1965; McKay, 2002; McKay, 2004; Summons et al., 2008; Shapiro R., 2009; Davies et al., 2009). More specifically, the principle that biological systems employ a discontinuous subset of the possible molecules in a family of biochemicals while abiotic syntheses produce a continuous spectrum of molecules, has been observed in many of these discussions and has been termed the "Lego Principle" by McKay (McKay, 2004, Davies et al., 2009). While indeed life uses only a subset of the possible molecules in many chemical families, a discontinous subset as a feature of life is not key to the argument we outline here. In at least some cases, as we will discuss later, the full spectrum of molecules are observed in both abiotic and biotic contexts. Thus it is only important to our argument that the abiotic and biotic abundance patterns be distinguishable, which we believe to be likely even in the case where all molecules in the family are observed in a sample.

We refer to the environmental distribution of molecular abundances as the "monomer abundance distribution biosignature" (MADB) and hypothesize that it is universal to all forms of life and collections of monomers they employ. While in principle we may observe such a biosignature in any set of chemical measurements, in practice it is easiest to demonstrate by comparing the relative concentrations of chemicals within a single family of related molecules 
(such as amino acids) where comparison of synthesis rates is arguably valid. If our hypothesis is true, it carries implications both for the understanding of evolving biochemistries and the detection of extraterrestrial life, where the biochemistry of a putative ecosphere is not $a$ priori known.

Of course, if significant periods of time elapse between the formation of the molecular distribution via biotic processes and its analysis, diagenesis can obscure any biosignature because different monomers may degrade at different rates. For example, the low molecular weight amino acids can come to predominate in natural sediments over time Abelson and Hare, 1969; Elster et al., 1991) simply because they are more stable, so that the original distribution is highly altered. The same is true of the signatures in meteorites, which must reflect the high-energy processing the organic compounds have experienced over the last 4.5 billion years.

While we apply the MADB to the challenge of detecting life in an extraterrestrial environmental sample, the use of monomer distributions as a diagnostic tool for other purposes is well-established. Fatty acid and phospholipid distributions have long been employed in the study of microbial diversity and other fields of biogeochemistry; see e.g. (White et al., 1997; Zelles and Bai, 1994; Sundh et al., 1997), and amino acid profiles are frequently used as indicators of the diagenetic state of organic matter (Hedges and Oades, 1997; Dauwe and Middelburg, 1998; Colombo et al., 1998). Amino acid profiles have also been used to distinguish between the synthesis pathways underlying abiotic amino acid formation in carbonaceous chondrites (Botta et al., 2002; Lerner et al., 1993). The relative frequencies of individual nucleotides in gene sequences are highly variable, but have statistical properties that can distinguish kingdoms and smaller families (Schultes et al., 1997; Gorban et al., 2003). Also, patterns of nucleic acids have been shown to reflect selective constraints such as RNA folding, thereby distinguishing functional from non-functional RNA (Schultes et al., 1999), as well as the likely availability of particular amino acids in the prebiotic environment (Brooks et al., 2002). Geochemical profiles have even been employed previously to detemine whether hydrocarbon compounds in Earth's mantle are of a biotic or abiotic source, through comparison to the contents of meteorites and known abiotic syntheses (Sugisaki and Mimura, 
1994).

Amino and carboxylic acids are familiar chemical families in which terrestrial biochemistry exhibits a clear signature of life's presence, and we collect and compare here data from a number of literature sources to demonstrate the effect. The statistical significance of the monomer abundance biosignature in amino acids has been previously studied (Dorn et al., 2003), and we review here the most salient results. In this study, we conduct a more thorough analysis of amino acids and include saturated monocarboxylic acids in order to demonstrate that the MADB phenomenon as observed in the terrestrial biosphere is not limited to only one monomer family.

Any proposed biosignature should be testable not only against terrestrial, but also extraterrestrial life. To quote Maynard Smith (1992): "So far, we have been able to study only one evolving system and we cannot wait for interstellar flight to provide us with a second. If we want to discover generalizations about evolving systems, we will have to look at artificial ones." We would like to go a little further to suggest that, in order to detect extraterrestrial life, we should have an understanding of the fundamental dynamics of living and evolving systems, independent of the organisms' particular substrate (i.e., chemistry). Digital life Adami, 1998; Ray, 1992) is an artificial form of life that provides just such an instance, and it has been used successfully as an experimental platform for the study of evolutionary dynamics Adami, 1998; Adami et al., 2000; Bell, 2001; Chow et al., 2004, Lenski et al., 1999; Lenski et al., 2003; Ofria et al., 2002; Ofria et al., 2003; Wilke and Adami, 2002; Wilke et al., 2001; Yedid and Bell, 2001). In a digital life system, self-replicating computer programs evolve within a digital ecosystem, enabling precise and controlled studies of evolutionary dynamics.

\section{The Avida Environment}

The Avida Digital Life Platform (Ofria and Wilke, 2004) is ideal for our purposes, since it is a controlled and understood system unrelated to terrestrial life. A digital system also makes possible repeated experimentation with evolution. An introduction to digital life can 
be found in Adami (1998) and Wilke and Adami (2002), while Adami (2006) should be consulted for a more recent overview of research conducted with this form of life.

In the digital world of Avida, computer programs of varying length encoded in a simple language replicate themselves and compete for processor time and physical space. Avidians evolve by mutation and selection of a self-replicating genome with access to a "chemistry" of simple different instructions (the monomers; 29 were available in the variant used for this study). Typically, a population is seeded with a primitive organism made from only 20 of those instructions. As the organism replicates, mutations (including copy errors, spontaneous point mutations, and insert and divide mutations) are imposed randomly on all organisms in the environment at rates configurable by the experimenter. Mutations and insertions create a new instruction (randomly chosen from the library of 29 with equal probability for each) at the target location.

In order to replicate, an avidian needs energy in the form of CPU (central processing unit) time. Each organism can increase its fraction of CPU time by performing calculations on a stream of random input numbers. These calculations (computational tasks) are the analogue of metabolic reactions (in biochemistry), and the code evolved to perform these tasks is analogous to the genetic code for metabolic enzymes. The simple self-replicator used as a progenitor (ancestor) organism does not include code to complete any mathematical tasks. As genotypes evolve computational genes, they receive a higher proportion of CPU time and quickly come to dominate the population.

We examined the relative distributions of computer instructions (the fundamental "monomers" in Avida) of numerous evolved and evolving populations to characterize the nature and robustness of the abundance patterns they form in response to evolutionary pressures. Avida organisms ("avidians") consist of a single genome: a string of computer instructions. These genomes evolve as a result of externally-imposed mutations and selection pressures (the environment). The computer instructions that compose avidians are analogous to the amino acids, fatty acids, or other compounds that compose familiar biota in the sense that they are selectable, meaning only those instructions that carry a fitness advantange will be repro- 
duced in future generations. Therefore, any general fitness advantage or adaptive utility of a single instruction should be visible in its bulk concentration in an evolved population.

We believe that Avida represents a sufficiently analogous model of biological evolution to test the MADB hypothesis. In both a biosphere and in the Avida simulator, self-replicating lifeforms are composed of one or more sets of monomers (e.g. amino acids, carboxylic acids, computer instructions). In the absence of life, abiotic formation processes will entirely determine the relative ratios of these monomers' concentrations, and we possess models and measurements of abiotic processes in both Avida and in organic chemistry. In the presence of life, however, the composition of the organisms will affect the concentration ratios of those monomers in the environment. In both Avida and the biosphere, competition will result in selection of the organisms, and this selection will act to optimize the composition of the organisms. For example, selection is unlikely to produce organisms whose proteins all comprise $95 \%$ glycine, since such proteins would not likely be functional, and certainly not optimally adapted. Likewise, in Avida, an adapted organism's instruction sequence is unlikely to contain $95 \%$ NOP-A instructions, since such a sequence would be inefficient at best. As a result, we can test in both the biosphere and in Avida the hypothesis that the concentration profile observed in an adapted organism - and by implication its environment - is unlikely to be the same we would observe in the absence of life.

In the biosphere, a high abundance of heavy amino acids like glutamate (relative to glycine) is diagnostic for life, because only a selected metabolism can account for such a thermodynamically improbable ratio. Likewise, in an Avida array, the presence of too-high or too-low concentrations of specific instructions can indicate the presence of selective pressure on the digital analogue of metabolism, since the Avida simulator inserts all instructions in equal proportion.

\title{
Materials and Methods
}

\author{
Terrestrial Biochemistry
}




\section{$\underline{\text { Data Sources }}$}

We compiled a database of measurements of related monomer concentrations (131 samples of amino acids, 31 samples of straight-chain monocarboxylic acids) from a variety of publications including studies of formation synthesis, terrestrial water columns and sediments, and meteorites.

Our set of abiotic amino acid data included thirty measurements in total, fifteen from tholin and spark-synthesis experiments (Khare et al., 1986, McDonald et al., 1994; McDonald et al., 1991; Miller and Urey, 1959; Ring et al., 1972; Schlesinger and Miller, 1986) one from a report of UV photolysis in deep-space conditions (Munoz-Caro et al., 2002), one of amino acid synthesis in a proton-irradiatiated mixture (Takano et al., 2004), seven from the Murchison meteorite Cronin et al., 1981; Cronin and Moore, 1976; Cronin et al., 1980; Cronin et al., 1979; Engel and Macko, 1997; Engel et al., 1990; Engel and Nagy, 1982; Kvenvolden et al., 1970) and six from two other carbonaceous chondrites (Cronin et al., 1979; Shimoyama et al., 1979). Based on composition and racemic mixture, the amino acids from these meteorites are generally agreed to be of abiotic origin (Cronin et al., 1994; Engel and Macko, 1997).

The complementary dataset of amino acids from the terrestrial biosphere included 125 measurements of extracts from a variety of terrestrial sediments, soils, and water columns Colombo et al., 1998; Cowie et al., 1992; Dauwe and Middelburg, 1998; Hedges et al., 2000; Horsfall and Wolff, 1997; Keil et al., 1998; Keil and Fogel, 2001; Kielland, 1995). These measurements include samples at a variety of ages and diagenetic condition ranging from fresh soil (Kielland, 1995) and river waters (Hedges et al., 2000) through ocean sediments up to 95 - 170 years old (Cowie et al., 1992).

Our database of carboxylic acids from abiotic sources includes two measurements from spark synthesis studies (Shimoyama et al., 1994; Yuen et al., 1981) and three from meteorite sources (Shimoyama et al., 1986; Naraoka et al., 1996; Lawless and Yuen, 1979). All five sources report only the concentrations of short-chain carboxylic acids (C12:0 and shorter). 
We rejected a few otherwise interesting measurements of amino acids including simulated Martian conditions (Abelson, 1965), early spark-synthesis studies (Miller, 1953), FischerTropsch type (FTT) and "thermal" syntheses (Anders et al., 1973; Hayatsu et al., 1971) as well as amber-encased insects (Wang et al., 1995) because they reported quantified measurements of too few of the amino acids (Gly, Ala, $\beta$-Ala, etc.) for useful comparison to other studies.

We compiled two different datasets of carboxylic acid measurements from terrestrial sources. We include only the straight-chain saturated monocarboxylic acids so that the data can be compared to sources of data on abiotic formation that report only this subfamily of compounds. The first dataset includes twelve measurements of the same low-weight acids as the abiotic dataset, all taken from coastal marine sediments (Shimoyama et al., 1991). A second group contains twenty-four measurements of longer chain carboxylic acids C14:0 through C28:0 in a variety of sediments and soils (Zelles and Bai, 1994; Wakeham, 1999; Colombo et al., 1996; Baath et al., 1992).

\section{Data Treatment}

To combine the disparate sources of data into unified sets for comparison, we applied the following protocol:

1. All data were converted to molar units.

2. Residues reported as "coeluted" (e.g., glycine and glutamate reported together as GLX) were both given the reported value.

3. "Trace" values were given a concentration of 0 .

4. Where enantiomers were reported separately, their sum was used in a combined column.

5. Values reported as "approximate" or "estimated" were used unchanged.

6. Where values were reported as " $<\mathrm{X}$ ", we used $\mathrm{X}$. 
It should be noted that while while enantiomeric excess has long been recognized as an important biosignature, we ignore it here because it was not measured in the vast majority of our data sources and is not the focus of this work.

In addition, we applied the following steps to the amino acid and long-chain carboxylic acid datasets:

7. Where a clear statement could be found in the text that a search was exhaustive (e.g., "all other residues were absent or present only at trace levels"), unreported measurements were assigned a zero.

8. Other unreported values were left blank and not included in averages.

9. Columns with more than $50 \%$ blank values in any one category were eliminated from the analysis. (e.g., Sarcosine is quantified in many spark-synthesis and meteorite studies, but never in sediment studies.)

The reduced amino acid database included eleven residues (from an initial thirty) that were quantified with sufficient frequency in both the biotic and abiotic categories: Gly, Ala, $\beta$ Ala, Aba, Ser, Ile, Leu, Asp, Glu, Thr, Val. The reduced long-chain carboxylic acid dataset included all straight-chain acids from C9:0 through C28:0, except C27:0. The abiotic component of the short-chain carboxylic acid dataset did not contain sufficient overlapping data for reasonable presentation of averages, so we have presented each measurement individually.

Each dataset was then normalized to a value appropriate for that category. All amino acid measurements were normalized to the mole concentration of glycine in that sample. The short- and long-chain carboxylic acid datasets were normalized to the concentrations of propionic acid (C3:0) and palmitic acid (C16:0), respectively.

Digital Life

$\underline{\text { Evolved Monomer Abundances in Terminal Populations }}$ 
Our first experiment examined the final distribution of instructions in 350 evolved populations that varied in physical parameters and initital conditions. In each run, we populated a grid of 3600 cells with one of two hand-coded ancestor genotypes, and allowed the population to evolve for 4000 generations. We quantified the bulk frequency of each of the instructions in the population every ten generations.

We used two different ancestor genotypes, (carefully written to differ as much as possible from each other), to control for the effect of initial conditions on the final evolved biosignature. The two progenitors were Avida's default 20-instruction ancestor, which uses the instructions SEARCH-F, JUMP-B and INC for the primary functions of flow control and replication, and an alternate 55-instruction ancestor that replaces those instructions with CALL, RETURN, SEARCH-B, SHIFT-L, DEC, and JUMP-F. Both organisms are shown in Table 1.

We also varied the copy-mutation rate in seven levels ranging by factors of two from 0.00125 to 0.08 mutations per copied instruction. At each combination of ancestor and mutation rate, we performed twenty-five runs that differed only in random seed.

Time-Evolution of a Biosignature

We conducted a second experiment in Avida to show the time-course of the evolving MADB as a formerly abiotic environment becomes dominated by incident life. We generated an abiotic population by initializing the same 3600-cell Avida grid with randomly-generated, nonviable (meaning nonreplicating) genomes. We created a lethal environment by bombarding the entire population with a high rate of point mutations. (A lethal mutation rate is so high that information cannot be maintained in a self-replicating genome; eventually the gene for self-replication is lost.) For the duration of the experiment, we periodically seeded this population with viable (mutation-free) progenitor organisms. This is analogous to a steady influx of space-borne spores onto a planet with a lethal environment and no existing biology. Then, we progressively stepped down the rate of point mutations until it reached a level where the organisms could survive and a population began to replicate and evolve. The population was allowed to evolve until the distribution of instructions had stabilized 
for several hundred generations. Then the point mutation rate was stepped back up until the population died and the randomizing effect of the mutations returned the instruction distribution to its "prebiotic" state.

\section{$\underline{\text { Data Treatment }}$}

Of the twenty-nine instructions in Avida's library, we exclude one instruction, NOP-A, from the analysis. NOP-A is used by the system to initialize empty memory in dividing organisms and is therefore greatly over-represented in comparison to the other instructions, and fluctuates significantly as organisms execute the ALLOCATE instruction. Furthermore, NOP-A's base rate of synthesis and availability is strongly genotype-dependent. It cannot be estimated a priori and we cannot make reasonable statements as to whether its abundance reflects biotic or abiotic processes, so we therefore compare only the remaining 28 instructions.

We quantified terminal abundances in evolved populations by averaging the last ten measurements, to provide data smoothing. Avidians in the process of replicating, and occasional nonviable mutations, cause small instantaneous fluctuations in population abundance of the individual instructions.

\section{Results}

\section{Terrestrial Biochemistry}

\section{$\underline{\text { Amino Acids }}$}

Glycine and alanine, which have low molecular weight and whose synthesis pathways are kinetically favorable, dominate mixtures of amino acids synthesized by abiotic processes. Some small non-protein amino acids, including sarcosine and beta-alanine, have been observed in synthesis experiments (Khare et al., 1986; McDonald et al., 1994, McDonald et al., 1991; Miller, 1955; Miller and Urey, 1959; Munoz-Caro et al., 2002; Schlesinger and Miller, 1986). Other amino acids are present only in trace concentrations, if at all McDonald et al., 1994; 
McDonald et al., 1991; Miller, 1955; Munoz-Caro et al., 2002). This pattern reflects the thermodynamics and kinetics of amino acid synthesis and is remarkably consistent regardless of the specific nature of the synthesis environment. For example, the alpha amino acids are likely formed via the Strecker (cyanohydrin) synthesis (Miller, 1955), which presumes the existence of precursor molecules that include the sidechain. The formation of heavier amino acids would require the prior formation of the larger sidechains: since these reactions would include their own kinetic barriers, the availability of the larger precursors and ultimately the final yields of the larger amino acids are expected to be lower. In addition, larger sidechains have a greater number of isomers. Therefore, unlike biotic synthesis where an enzyme catalyzes formation of a specific desired isomer, we would expect an abiotic synthesis to produce smaller quantities of many isomers.

Some of the heavier protein amino acids such as Tyr and His have been detected in FTT and high-temperature syntheses (Anders et al., 1973; Hayatsu et al., 1971). However, we are unaware of any measurements of these molecules in abiotic synthesis in quantities that approach the concentration of Gly. Phe and Thr are not seen in abiotic synthesis.

Amino acids in terrestrial samples show a more varied distribution dominated by the protein amino acids in roughly equal proportions. Figure 1 shows the relative abundances of a set of twelve amino acids measured in a variety of biotic and abiotic samples. The amino acids are plotted in ascending order of $\Delta \mathrm{G}_{r}$ (Gibbs free energy of synthesis) at $18^{\circ} \mathrm{C}$ as reported by Amend and Shock (1998) to show both the trend among abiotic synthesis products towards smaller and "simpler" compounds and the biosynthesis of complex, expensive compounds. The "Sediment" curve represents terrestrial sediment and water column extractions. "Meteorite" represents extractions from three carbonaceous chondrites, and "Synthesis" is an average of fifteen spark-synthesis experiments in a variety of atmospheres and a single extraction from amino acids synthesized via UV photolysis on analogues of interstellar ice. The Synthesis curve serves as an abiotic baseline: the pattern of amino acid concentrations we expect when life is not present. Except for a single anomaly (high glutamate concentration), the meteorite curve exhibits the same pattern, while the biologically-generated Sediment curve differs significantly, exhibiting high concentrations of a number of the larger amino 


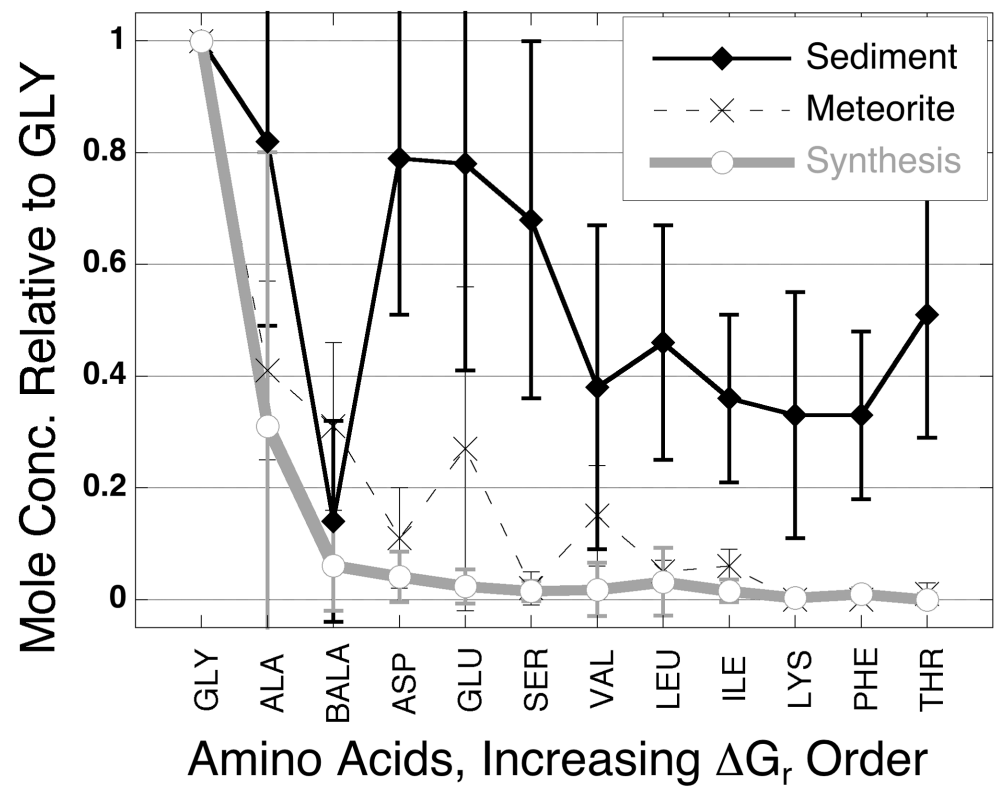

Figure 1: Average patterns of amino acid abundances relative to glycine, compared between biotic (Sediment $n=125$ ) and abiotic (Meteorite $n=15$, Synthesis $n=16$ ) sources. Error bars are one standard deviation.

acids. The measured concentrations of the heavier protein amino acids vary greatly in biotic samples, but are consistently higher than is ever observed in abiotic sources.

This analysis only shows a small selection out of hundreds of possible amino acids. A quantification of all possible low-molecular-weight amino acids would show several, such as sarcosine, that are non-zero in the abiotic baseline but low or zero in the biotic curves, similar to beta-alanine in Figure 1. Many heavier amino acids would not be present in either case. Therefore, the complete amino acid biosignature for terrestrial organisms would appear graphically as a few dozen peaks for the protein amino acids, other biologically important amino acids such as synthesis intermediates and neurotransmitters, and major breakdown products in a large spectrum of hundreds of possible, but absent, amino acids. This sparse subset and its utility as a biosignature is referred to by McKay as the Lego Principle (McKay, 2004). Fig. 1 is a subset of the hypothetical biosignature plot that includes all possible amino acids found in either biotic or abiotic contexts.

Carboxylic Acids 
Preparations from spark-synthesis studies exhibit carboxylic acids of low carbon number: only acids C6:0 and smaller are seen in significant quantities, and acids larger than C12:0 are not found at all in most experiments (Shimoyama et al., 1994; Yuen et al., 1981). Figure 2 shows the relative concentrations of the first few acids measured in two such experiments and as measured in three meteorites, compared to an average of twelve measurements of terrestrial sediments. We observe in the Synthesis data a consistent exponential decrease in concentration with increasing molecular weight, while the Sediment curve shows a much more even distribution. The meteorite data fall between the two extremes, with a shallower exponential decrease and some variability in the relative concentration of acetic acid, probably due to its relatively high volatility and possible outgassing in space or during atmospheric entry. We contend that the pattern reflects the same fundamental processes that are at work in the amino acid data. As molecules become larger, formation energies are higher, synthesis kinetics generally become less favorable, and the number of possible conformations is greater, reducing the expected relative concentration of any particular conformer. The data from the meteorites implies a probable abiotic origin with a small amount of contamination by terrestrial material. We are not aware of any significant quantities of carboxylic acids larger than C12:0 recovered from non-contaminated meteorites.

Long-chain (up to C33) lipid compounds have been created in Fischer-Tropsch type (FTT) syntheses at high $\left(200{ }^{\circ} \mathrm{C}\right.$ and greater $)$ temperatures, but such experiments report no bias toward any particular carbon number (McCollom et al., 1999; Rushdi and Simoneit, 2001). Therefore the bias towards even-numbered carbon chains in carboxylic acids serves as an MADB even when long-chain-producing FTT processes are included in the abiotic baseline.

In terrestrial samples, however, the higher molecular weight acids C14:0 through C32:0 are present in abundance. As shown in Figure 3, environmental samples exhibit a consistent pattern that includes a primary peak at C16:0 with smaller peaks favoring even-carbon-number acids. This pattern cannot be explained by formation thermodynamics, but rather reflects a pair of biological constraints. A fitness criterion is present: carbon chain lengths near 16, in both single- and double-chain amphiphiles, are ideal for the formation of stable, non-porous, and flexible bilayers in aqueous solution at biologically relevant ranges of temperature and $\mathrm{pH}$ 


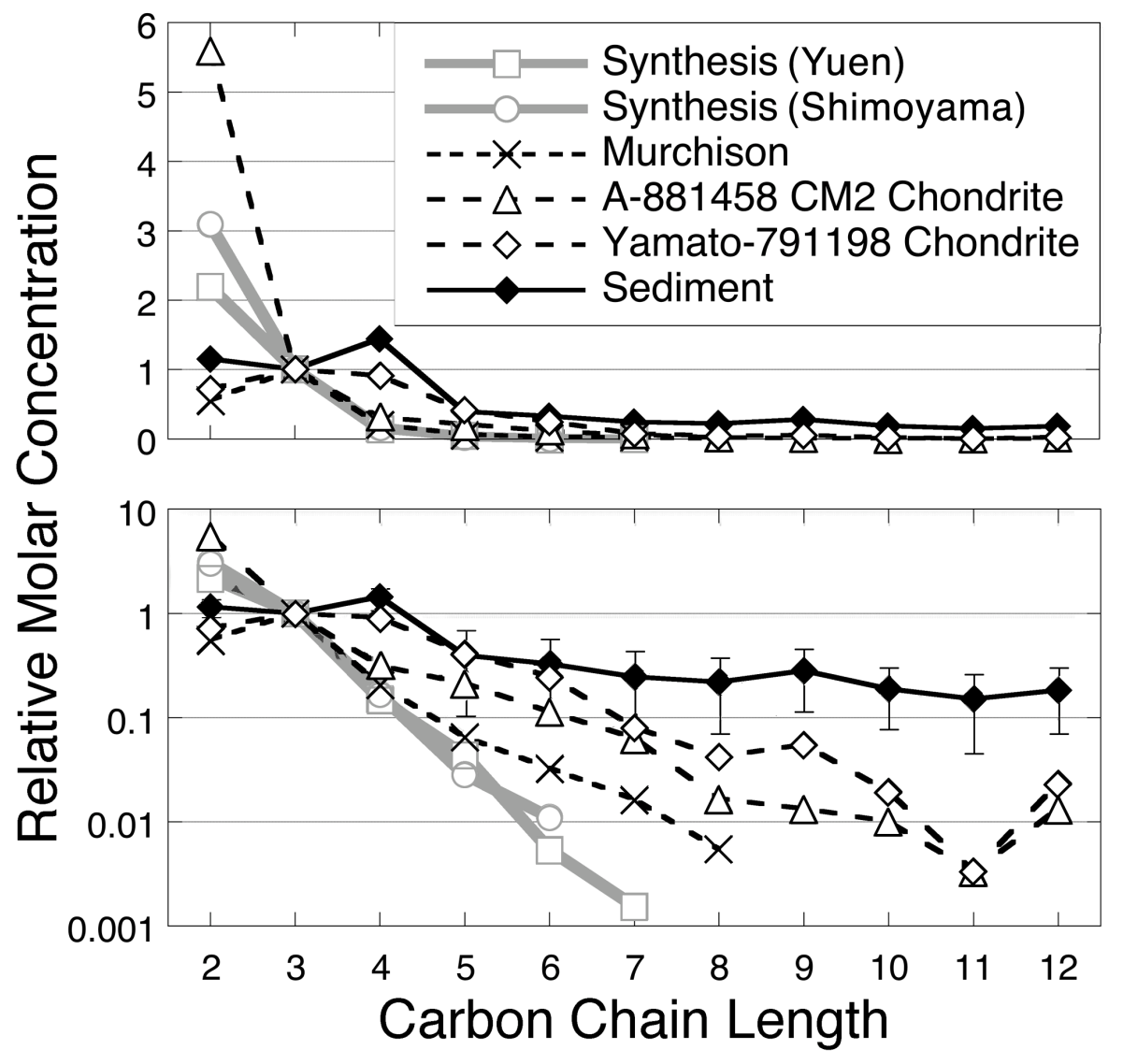

Figure 2: Relative concentrations of low-carbon number monocarboxylic acids from five abiotic sources compared to the average of 12 measurements in sediments. The data are plotted again on a semilog scale below to show the trend of decreasing concentration after C6:0 in abiotic samples. Synthesis curves represent two individual experiments (Shimoyama et al. 1994; Yuen et al. 1991). 


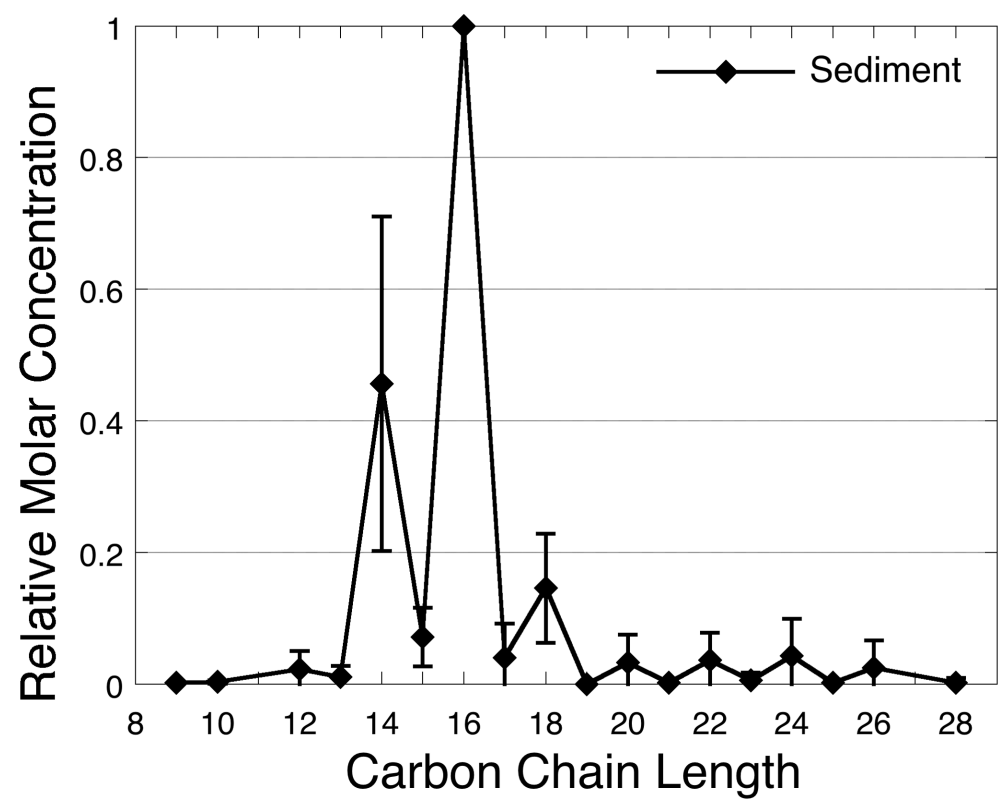

Figure 3: Curve representing the average pattern of long-chain monocarboxylic acids found in terrestrial sediments $(n=26)$, showing an apparent bias towards even-numbered carbon chains not seen in abiotic syntheses.

(Hargreaves and Deamer, 1978; Deamer, 1999; Gebicki and Hicks, 1976), and are therefore critical for membrane structure in a water-based biosphere. The predominance of evennumbered carbon chains reflects processes specific to terrestrial biochemistry: the primary fatty acid biosynthesis cycle terminates at C16:0 (palmitate), from which other fatty acids are synthesized through lengthening or shortening cycles that operate in two-carbon steps. A bias toward even-numbered carbon chains is considered diagnostic of biosynthesis and has been used to identify terrestrial contamination in meteorite sources (Nagy and Bitz, 1963). The slight increase in concentration of C12:0 seen in two of the meteorites in Fig. 2 probably represents low-level terrestrial contamination.

Because all carboxylic acids from C2 through C28 are found in measurable concentrations in both biotic context and in abiotic context (when FTT syntheses are included), the Lego Principle (McKay, 2004) - that biological samples include only a subset of the possible molecules in a family - is not observed in monocarboxylic acids. However, because the shapes of the abundance curves are so different in the two contexts, an MADB is still observable in 
the sense originally described by Lovelock (Lovelock, 1965).

\section{Digital Life}

$\underline{\text { Monomer Abundances in Terminal Populations }}$

In Avida, two processes are present that we may consider "abiotic." First, we initalize the population with a hand-written, progenitor genotype. Second, mutations in Avida result in the replacement of an instruction with a new one, randomly chosen from the library with equal probability for each possible instruction. If selection did not affect the distribution of instructions, we would expect one or both of these effects to constrain the evolved abundance. If the initial conditions imposed by the ancestor constrained the evolved population, we would expect the final abundance to reflect the ancestor's distribution. If mutation were the dominant effect, as we would expect over long periods of time, the final population would show each of the instructions in equal proportion, demonstrating that the genotypes had incorporated the instructions at the same rate with that they appreared in mutation. Thus, the abiotic expectation in Avida, analogous to the Synthesis curves of Figs. 1 and 2, is a flat line.

We observe in nearly all Avida runs, regardless of initial conditions or variables, a specific and consistent pattern of evolved instruction abundance that reflects neither of the abiotic constraints as a dominant feature. Figures $4 \mathrm{a}$ and $4 \mathrm{~b}$ compare bulk instruction frequency curves to the abiotic expectation for populations from the two progenitor organisms in Table 1. Experiments were conducted at a number of different mutation rates, where $\mu$ represents the probability that an instruction is mutated when it is being copied into a progenitor's genome (the "per-site copy mutation" rate). While some variability is present as conditions change, many of the general features of the pattern are conserved and all evolved patterns are distinguishable from the abiotic baseline. The relative abundances observed in our experiments differ not only in their mean, but also in their variance. Instructions critical to an organism's survival (such as COPY and DIVIDE) are usually present at predictable levels (here, one per genome), and therefore show little variability. Optional instructions such as 


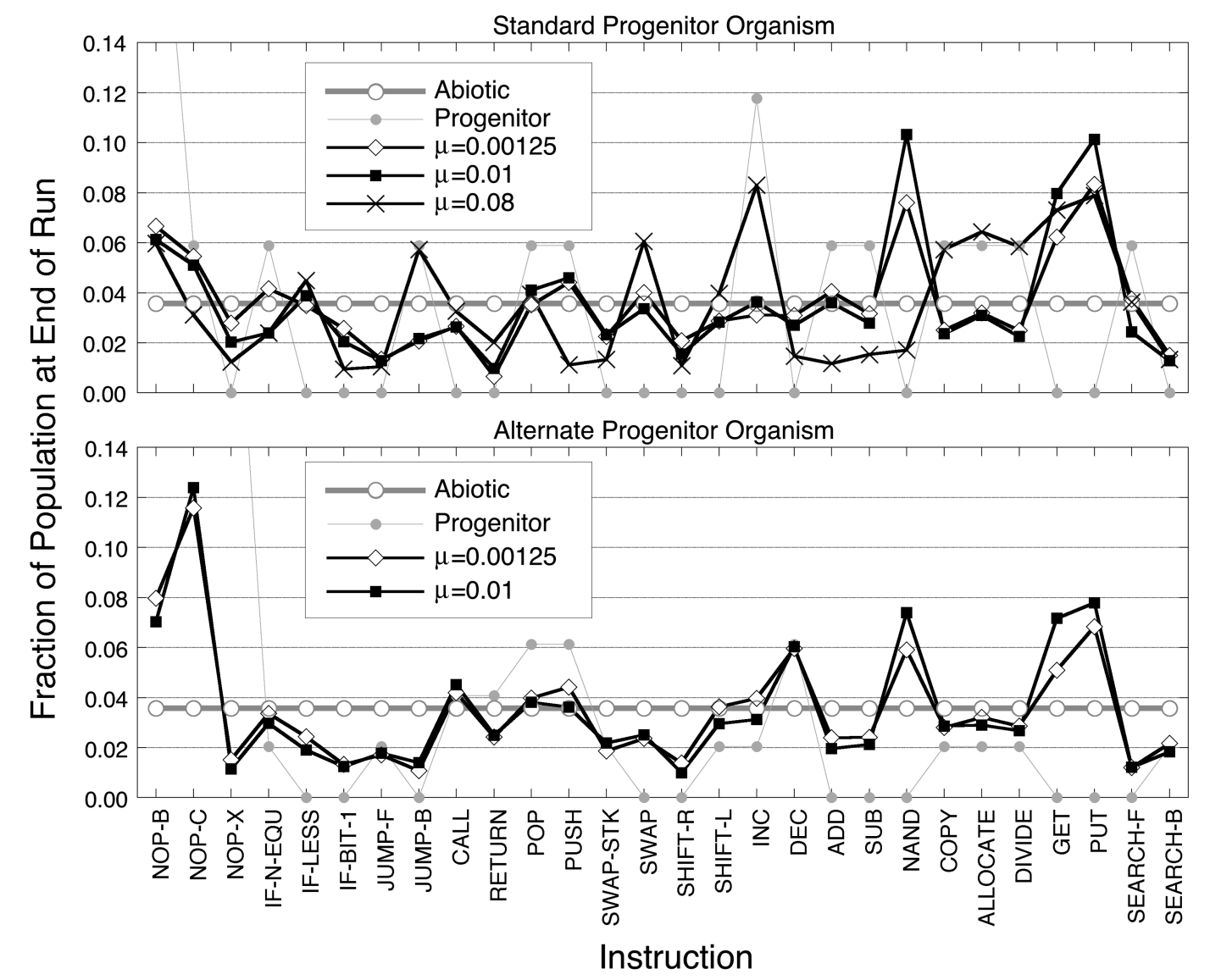

Figure 4: Relative abundance curves for several evolved populations. Note how a wide range of mutation rates ( $\mu=0.00125$ to 0.01 ) produces nearly identical distributions. Tests at numerous intermediate values of $\mu$ showed similar results (not shown). Near-lethal mutation rates $(\mu=0.08)$ and alternate progenitor organisms produce distinct distributions that share some features with the "standard" distribution. $n=25$ for each curve.

NAND, on the other hand, are expressed at high but variable concentrations as organisms in different competitive environments use them to complete different mathematical tasks (see "The Avida Environment" in Materials and Methods). Instructions that are frequently lethal when they appear as mutations (e.g., RETURN and JUMP-F) are suppressed, and the useless but non-fatal instruction NOP-X appears at a low but persistent level.

The distribution of relative abundances is essentially consistent over a large range of mutation rates (factor of 32), and changes only as the mutation rate becomes so high that the majority of offspring contain at least one mutation. At a near-lethal mutation rate of $\mu=0.08$, 


\begin{tabular}{|c|c|c|c|c|}
\hline Progenitor & \multicolumn{2}{|c|}{ Alternate Progenitor } & \multicolumn{2}{|c|}{ Typical Evolved Avidian } \\
\hline 1 SEARCH-F & $1 \mathrm{CALL}$ & $29 \mathrm{DEC}$ & 1 SEARCH-F & 29 NOP-C \\
\hline 2 NOP-A & 2 NOP-A & 30 NOP-C & 2 NOP-A & $30 \mathrm{PUT}$ \\
\hline 3 NOP-A & 3 NOP-A & 37255 & 3 NOP-A & 31 NOP-X \\
\hline $4 \mathrm{ADD}$ & 4 SHIFT-L & 32 NOP-C & $4 \mathrm{ADD}$ & 32 DIVIDE \\
\hline 5 INC & 5 ALLOCATE & $33 \mathrm{PUSH}$ & $5 \mathrm{ADD}$ & 33 PUT \\
\hline 6 ALLOCATE & $6 \mathrm{PUSH}$ & 34 NOP-C & $6 \mathrm{GET}$ & 34 SHIFT-R \\
\hline $7 \mathrm{PUSH}$ & 7 SWAP-STK & 35 RETURN & 7 ALLOCATE & $35 \mathrm{DEC}$ \\
\hline 8 NOP-B & $8 \mathrm{PUSH}$ & 36 NOP-X & $8 \mathrm{NAND}$ & $36 \mathrm{PUT}$ \\
\hline $9 \mathrm{POP}$ & 9 NOP-B & 37 NOP-X & $9 \mathrm{PUT}$ & 37 NOP-B \\
\hline 10 NOP-C & $10 \mathrm{POP}$ & 38 NOP-X & 10 NOP-C & $38 \mathrm{COPY}$ \\
\hline $11 \mathrm{SUB}$ & 11 NOP-C & 39 NOP-X & $11 \mathrm{PUT}$ & 39 INC \\
\hline 12 NOP-B & $12 \mathrm{POP}$ & 40 NOP-X & 12 NAND & 40 IF-N-EQU \\
\hline $13 \mathrm{COPY}$ & 13 NOP-B & 41 NOP-X & $13 \mathrm{GET}$ & 41 NOP-A \\
\hline $14 \mathrm{INC}$ & 14 IF-N-EQU & 42 NOP-X & 14 NAND & 42 JUMP-B \\
\hline 15 IF-N-EQU & 15 NOP-A & 43 NOP-X & $15 \mathrm{GET}$ & 43 NOP-A \\
\hline 16 JUMP-B & 16 CALL & 44 NOP-X & 16 PUSH & $44 \mathrm{GET}$ \\
\hline 17 NOP-A & 17 NOP-A & 45 NOP-X & $17 \mathrm{PUT}$ & 45 NOP-B \\
\hline 18 DIVIDE & 18 NOP-B & 46 NOP-X & $18 \mathrm{POP}$ & 46 NOP-B \\
\hline 19 NOP-B & 19 DIVIDE & 47 NOP-B & 19 NOP-X & 47 JUMP-B \\
\hline 20 NOP-B & 20 JUMP-F & 48 NOP-B & 20 IF-N-EQU & \\
\hline & 21 NOP-B & 49 SEARCH-B & 21 NAND & \\
\hline & 22 NOP-C & 50 NOP-A & 22 IF-BIT-1 & \\
\hline & $23 \mathrm{COPY}$ & 51 NOP-B & 23 NAND & \\
\hline & $24 \mathrm{INC}$ & 52 RETURN & $24 \mathrm{GET}$ & \\
\hline & $25 \mathrm{POP}$ & 53 NOP-X & 25 NAND & \\
\hline & 26 NOP-C & 54 NOP-C & 26 NAND & \\
\hline & $27 \mathrm{DEC}$ & 55 NOP-A & 27 NOP-C & \\
\hline & 28 NOP-C & & $28 \mathrm{PUT}$ & \\
\hline
\end{tabular}

Table 1: Examples of three Avida organisms. The progenitors, used to seed initial populations, were hand-coded and are poorly adapted to the Avida environment. The "evolved avidian" is an example genome that arose approximately 1000 generations into a typical run seeded with the standard progenitor. 
a change in survival strategy generates a dramatic shift in the biosignature. Organisms abandon mathematical tasks and opt for very short genomes in order to maximize the likelihood of spawning genetically pure offspring. Mathematics-related instructions (NAND) are suppressed, while instructions that generally only appear once per genome (COPY, ALLOCATE, DIVIDE) are present at a higher concentration in the population as a whole because of the shorter average genome length. Other features, such as suppression of often-lethal mutations (JUMP-F, RETURN) are retained.

The observed pattern of evolved instruction abundance retains most of its salient features when we seed the population with a drastically different progenitor organism (See Table 1), demonstrating that the constraints of adaptation generally overwhelm the effect of initial conditions. As runs progress, the signatures of populations descending from the two disparate progenitors tend to converge, as some features are conserved among well-adapted populations. Most populations tend to express high frequencies of NAND, since it is essential for solving mathematical tasks and therefore high fitness.

There are some features of the abundance profile that Avidians tend to retain from their ancestor. For example, descendants of the standard progenitor tend to use JUMP-B (jump backward) for loop construction, while descendants of the alternate progenitor tend to use CALL/RETURN (call subroutine and return from subroutine) for the same basic function. Likewise one clade tends to use decrementing loop counters with DEC while the other uses INC. These local fitness maxima represent distinct genotypes that are fairly well adapted themselves but cannot be derived from each other via evolution because the intermediate mutations required to convert one genotype into the other would be invariably fatal. For example, the two progenitors differ in implementing their essential "copy loop": one uses the INC (increment) operator while the other uses DEC (decrement). Changing INC to DEC in the copy loop would require several simultaneous mutations, any one of which alone would be fatal. As a result, this transformation is never seen in practice. Though INC and DEC may serve other functions in an organism, evolved populations tend to express the operator used by their ancestor's copy loop. This effect is evident in Fig. 4. 


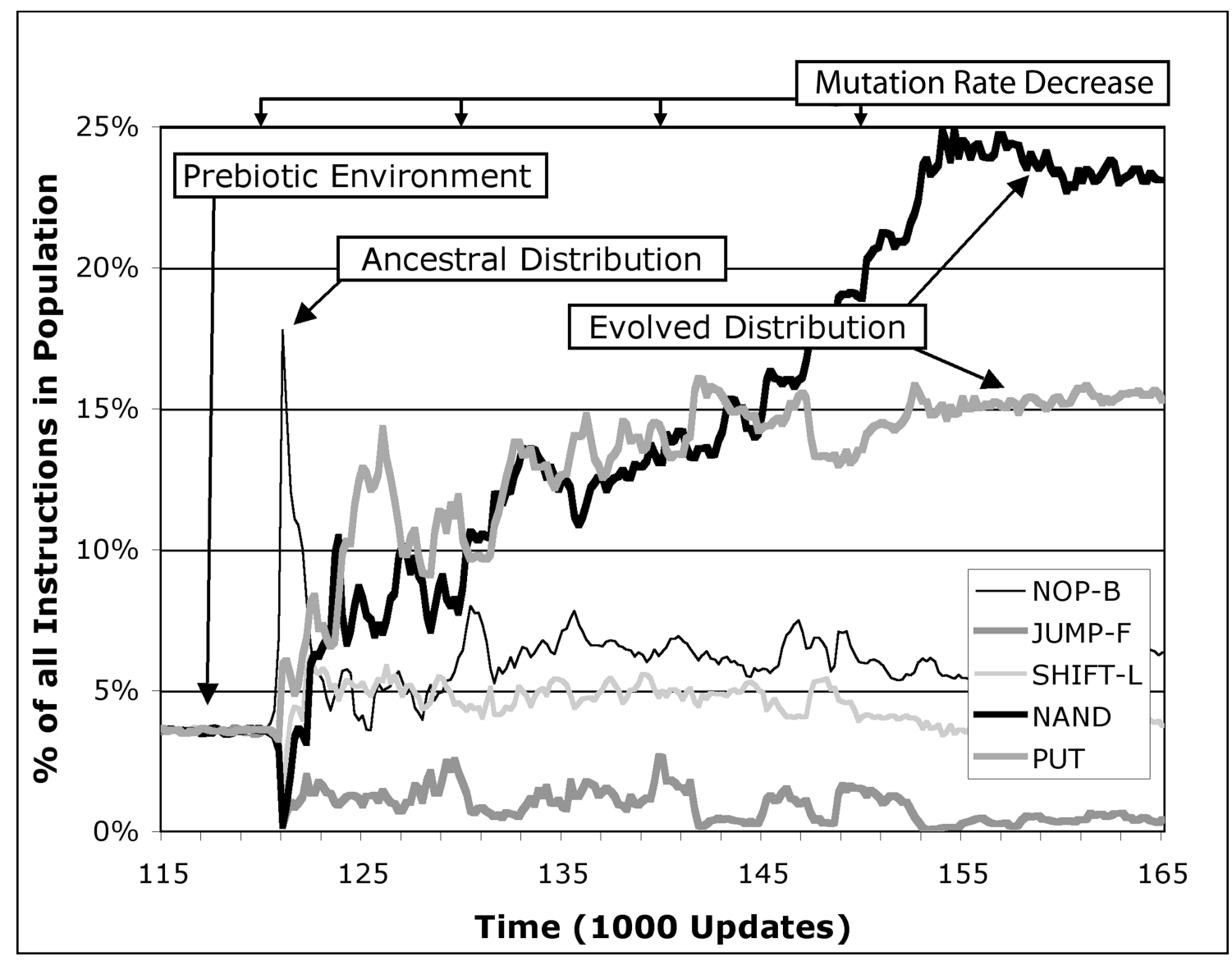

Figure 5: Abundance vs. time for six instructions as an incident lifeform populates a previously abiotic environment. The ancestor's most abundant instruction is NOP-B, while the adapted population's most abundant instruction is NAND. All instructions were present in equal proportion in the initial, mutation-dominated environment that did not contain any replicating organisms. A single standard Avida ancestor was seeded into the population every 200 updates. Point mutation ("cosmic ray") rate was stepped down progressively every 10,000 updates from the start of the experiment until update 150,000. At 120,000 updates the mutation rate dropped from the lethal level of $2.67 \times 10^{-3}$ mutations per update per site to a nonlethal level of $2.00 \times 10^{-3}$ mutations per update per site. After update 120,000 , one of the ancestors was able to replicate and establish a population before being destroyed by point mutations. 
Time-Evolution of a Biosignature

When viable self-replicators begin to dominate a previously lifeless Avida grid, their signature frequency of instructions quickly overwhelms the pre-existing random distribution. Figure 5 shows the time-varying abundances of several instructions in one such run where sequences capable of self-replication were consistently re-injected into the population at a small rate, while sequences were bombarded with a high rate of point mutations per-site that is independent of the copy-process of replication, that is, the mutations were of the "cosmic ray" type. At the outset, all instructions were present in equal abundance as the cells contained randomized, nonviable code because the injected replicators could not take hold. This gives rise to exactly the hypothetical abundance pattern used as an abiotic baseline in Fig. 4. When the point mutation rate was decreased to a non-lethal level, replication immediately became the dominant process. As the population of replicators filled the available space, the distribution of instructions in the ancestor's genome began to dominate, but was quickly replaced as higher-fitness organisms evolved. A recognizable distribution evolves with the same features we see in Fig. 4, such as high NAND concentration but suppressed JUMPF. The distribution stabilizes after a few hundred generations, even though the organisms themselves were still evolving.

We tracked all 28 instructions and allowed the population to evolve for several thousand generations beyond the point shown in Fig. 5. Eventually, we increased the mutation rate to its previous level, and the population died. At that point, the bombardment of point mutations rapidly returned the environment to its prebiotic state. Digital movie files showing the time evolution of all 28 instructions for the full time course of the experiment shown in Fig. 5., and the development of one run used to generate Fig. 4, may be found in the online supplemental information.

\section{Discussion}

We believe the monomer abundance distribution biosignature is a universal feature of evolv- 
ing systems: rapid synthesis of a select subset of compounds by a living metabolism should inevitably leave a biosphere in notable disequilibrium with respect to a sterile planet of the same geochemical composition. If we consider any particular monomer family, it is unlikely that thermodynamics would constrain abiotic synthesis to precisely the same rates as biotic synthesis. Indeed, if abiotic synthesis did produce monomers in the same concentrations as evolved biosynthesis, then biosynthesis would not be necessary, selection could not improve on abiotic geochemistry, and we question whether such a circumstance could in fact be described as "life."

The "lock-in" effect where some features of the ancestral avidian survive despite the availability of equivalent instructions is analogous to the persistent use by terrestrial biology of a subset of the amino acids, selected early in life's evolution, that have complementary properties. For example, it is essential that we have amino acids with both hydrophilic and hydrophobic side-chains, but it may not have been necessary that life uses exactly the sets we have. The possibility of early life having being initiated with a different set of amino acids that nonetheless span the necessary and complementary functions is analogous to the early lock-in of CALL/RETURN rather than JUMP-B for looping.

Furthermore, the MADB should be detectable whenever life is present. While both abiotic and biotic syntheses may be present in an environment, we may reasonably expect biosynthesis to dominate most molecular synthesis as it does in the terrestrial biosphere. The coupling of energy from metabolism to catabolism, the manufacture of catalysts (enzymes), and the ability to concentrate reactants inside cells conspire to generate vast increases in synthesis rates relative to abiotic chemistry. As a result, even small quantities of extant life should leave a detectable chemical signature.

Understanding evolution's effects on the chemical environment resulting from selection operating on metabolizing organisms carries significance both for our general understanding of life and for the search for extraterrestrial life. It is essential for the latter because we cannot predict the specific biochemistry of the putative life for which we are searching. A hypothetical extraterrestrial biochemistry might use a different set of amino acids, or use them in 
different abundances. But while such a pattern may not look like the terrestrial data in Fig. 1, it would be unlikely to match the consistent pattern seen in abiotic syntheses. Likewise, extraterrestrial fatty acid biosynthesis could conceivably use cycles that operated in one- or three-carbon increments, resulting in a different distribution of peaks. If the organisms used ammonia, methane, or some other primary solvent rather than water, the adaptive peak for stable membranes might not be C:16. In either case, the unique signature of this exotic biochemistry would reflect the adaptive constraints of these organisms' biosphere and could be very unlike the "Synthesis" curves in Figure 2.

It is of course also possible that an extraterrestrial biosphere might not employ aminoand carboxylic acids at all. Although some argue that the same families of molecules seen in terrestrial biology (proteins, fats, sugars, nucleic acids, etc.) are likely to be found in carbon-based life everywhere (Pace, 2001), an unbiased search should include the possibility of life using alternate chemistry. Doing so would mean generating thorough abiotic profiles of a wide variety of chemical families, and equipping a probe or measurement device with the ability to assay numerous compounds in each family to detect abundance profiles deviating from the range of abiotic measurements.

A contained digital system like Avida has several advantages for studying the MADB hypothesis. The course of evolution can be studied repeatedly to provide statistical significance to dynamics that usually only yield singular, history-dependent event. With terrestrial life, we can only examine one example of an evolved biochemistry: we cannot "start life over" on Earth and measure the concentrations of amino or carboxylic acids in each resulting biosphere, and therefore cannot prove that the particular concentration patterns we observe are the result of evolutionary selection's effect on biochemistry rather than happenstance. In Avida, we can repeat the process indefinitely to obtain statistics about how organisms adapt. In addition, we have complete control over initial conditions, allowing us to accurately characterize their impact on the result. Finally, Avida implements the replication and evolution aspects of life in a system otherwise completely unrelated to terrestrial biochemistry, giving us an opportunity to study a biosignature protocol in a Earth-independent setting. 


\section{Conclusions}

The relative distribution of abundances of a set of monomers is a strong biosignature that may show promise for detecting alien biochemistries where sufficient population exists to imprint a chemical signature. The constraints of adaptation, combined with the growth of biomass, overwhelm the abiotic chemistry present in the environment as organisms expend energy to build complex, low-entropy structures. The similarity between laboratory synthesis and meteorite samples is remarkable since meteorite samples may have formed under conditions very different from laboratory syntheses and have aged under planetary and/or deep space conditions for thousands or millions of years before collection and analysis.

Using the digital life platform Avida, we have demonstrated that the monomer abundance biosignature phenomenon may be germane to any living system, and have studied the robustness of the marker across a range of mutation rates and seed organisms. While an exhaustive analysis of initial conditions cannot be performed for this simple model life-form, the robustness of discrimination we see given the variety of evolutionary paths and organisms produced in these experiments bodes well for the universality and applicability of the MADB.

It may be possible to look for instances of the monomer abundance biosignature simply by

empowering a life-detection system with a few basic models (derived theoretically and/or empirically) of expected abiotic chemical abundances, and then examining samples for patterns that differ significantly. Strong deviations from thermodynamic/kinetic expectations, i.e., the appearance of unusual concentrations of any high formation energy, low probability compounds, could signal that a site or sample may contain life and is worthy of further investigation. This strategy poses significant challenges, but ultimately frees the search for extraterrestrial life from biases centered in our present understanding of terrestrial biochemisty.

Creating accurate models of expected conditions will be difficult, as unfamiliar local effects and boundary conditions (e.g., excessive concentrations of a certain mineral, or unusual tem- 
peratures) could change the chemical species present in a sample and cause a false positive. However, we may find some safe generalizations (for example, that lysine, arginine, and histidine will always be absent or low in the absence of life) that enable astrobiologists to build reliable experiments.

\section{Acknowledgements}

The research described in this work was carried out in part at the Jet Propulsion Laboratory, California Institute of Technology, under a contract with the National Aeronautics and Space Administration (NASA), with support from the Director's Research and Development Fund (DRDF) and from the National Science Foundation under contract No. DEB-9981397. We thank Claus Wilke, Ronald V. Dorn III, and Diana Sherman for discussions. Finally, we are grateful to three anonymous reviewers for extensive and constructive comments on the manuscript.

\section{References}

Abelson PH (1965) Abiogenic synthesis in martian environment. Proc Natl Acad Sci U S A 54:1490-1494

Abelson PH, Hare PE (1969) Recent amino acids in the gunflint chert. Carnegie Inst Washington Yearbook 69:208-210

Adami C (1998) Introduction to Artificial Life. Springer, New York

Adami C (2006) Digital genetics: unravelling the genetic basis of evolution. Nature Reviews Genetics 7:109-118

Adami C, Ofria C, Collier TC (2000) Evolution of biological complexity. Proc Natl Acad Sci USA 97:4463-4468 
Amend JP, Shock EL (1998) Energetics of amino acid synthesis in hydrothermal ecosystems. Science 281:1659-1662

Anders E, Hayatsu R, H SM (1973) Organic compounds in meteorites. Science 182:781-790

Baath E, Frostegard A, Fritze H (1992) Soil bacterial biomass, activity, phospholipid fattyacid pattern, and ph tolerance in an area polluted with alkaline dust deposition. Appl Environ Microbiol 58:4026-4031

Bell G (2001) Neutral macroecology. Science 293:2413-2418

Botta O, Glavin DP, Kminek G, Bada JL (2002) Relative amino acid concentrations as a signature for parent body processes of carbonaceous chondrites. Origins Life Evol Biosph 32:143-163

Brooks DJ, Fresco JR, Lesk AM, Singh M (2002) Evolution of amino acid frequencies in proteins over deep time: Inferred order of introduction of amino acids into the genetic code. Mol Biol Evol 19:1645-1655

Chow SS, Wilke CO, Ofria C, Lenski RE, Adami C (2004) Adaptive radiation from resource competition in digital organisms. Science 305:84-86

Colombo JC, Silverberg N, Gearing JN (1996) Lipid biogeochemistry in the Laurentian trough .1. Fatty acids, sterols and aliphatic hydrocarbons in rapidly settling particles. Org Geochem 25:211-225

Colombo JC, Silverberg N, Gearing JN (1998) Amino acid biogeochemistry in the laurentian trough: vertical fluxes and individual reactivity during early diagenesis. Org Geochem 29:933-945

Cowie GL, Hedges JI, Calvert SE (1992) Sources and relative reactivities of amino-acids, neutral sugars, and lignin in an intermittently anoxic marine- environment. Geochim Cosmochim Acta 56:1963-1978

Cronin JR, Cooper GW, Pizzarello S (1994) Characteristics and formation of amino-acids and hydroxy-acids of the Murchison meteorite. In: Life Sciences and Space Research 
XXV (4), Pergamon Press Ltd., Oxford, Vol. 15 of Advances in Space Research, pp. 91-97

Cronin JR, Gandy WE, Pizzarello S (1981) Amino-acids of the Murchison meteorite .1. 6 carbon acyclic primary alpha-amino alkanoic acids. J Mol Evol 17:265-272

Cronin JR, Moore CB (1976) Amino-acids of Nogoya and Mokoia carbonaceous chondrites. Geochim Cosmochim Acta 40:853-857

Cronin JR, Moore CB, Pizzarello S (1980) Amino-acids in six CM2 chondrites. Meteoritics $15: 277-278$

Cronin JR, Pizzarello S, Moore CB (1979) Amino-acids in an antarctic carbonaceous chondrite. Science 206:335-337

Dauwe B, Middelburg JJ (1998) Amino acids and hexosamines as indicators of organic matter degradation state in north sea sediments. Limnol Oceanogr 43:782-798

Davies PCW, Benner SA, Cleland CE, Lineweaver CH, McKay CP, Wolfe-Simon F (2009) Signatures of a shadow biosphere. Astrobiology 9:241-249

Deamer DW (1999) How did it all begin? The self-assembly of organic molecules and the origin of cellular life. In: Scotchmoor J, Springer DA (Eds.) Evolution: Investigating the Evidence, The Paleontological Society, Knoxville, Vol. 9

Dorn ED, McDonald GD, Storrie-Lombardi MC, Nealson KH (2003) Principal component analysis and neural networks for detection of amino acid biosignatures. Icarus 166:403409

Elster H, Emanuel G, Weiner S (1991) Amino acid racemization of fossil bone. Journal of Archaeological Science 18:605-617

Engel MH, Macko SA (1997) Isotopic evidence for extraterrestrial non-racemic amino acids in the Murchison meteorite. Nature 389:265-268

Engel MH, Macko SA, Silfer JA (1990) Carbon isotope composition of individual amino-acids in the Murchison meteorite. Nature 348:47-49 
Engel MH, Nagy B (1982) Distribution and enantiomeric composition of amino-acids in the Murchison meteorite. Nature 296:837-840

Gebicki JM, Hicks M (1976) Preparation and properties of vesicles enclosed by fatty acid membranes. Chem Phys Lipids 16:142-160

Gorban AN, Zinovyev AY, Popova TG (2003) Seven clusters in genomic triplet distributions. In Silico Biol 3:471-482

Hargreaves WR, Deamer DW (1978) Liposomes from ionic, single-chain amphiphiles. Biochemistry 17

Hayatsu R, H SM, Anders E (1971) Origin of organic matter in early solar system - iv. Amino acids: Confirmation of catalytic synthesis by mass spectrometry. Geochim Cosmochim Acta 35:939-951

Hedges JI, Mayorga E, Tsamakis E, McClain ME, Aufdenkampe A, Quay P, Richey JE, Benner R, Opsahl S, Black B, Pimentel T, Quintanilla J, Maurice L (2000) Organic matter in Bolivian tributaries of the Amazon River: A comparison to the lower mainstream. Limnol Oceanogr 45:1449-1466

Hedges JI, Oades JM (1997) Comparative organic geochemistries of soils and marine sediments. Org Geochem 27:319-361

Horsfall IM, Wolff GA (1997) Hydrolysable amino acids in sediments from the Porcupine Abyssal Plain, northeast Atlantic Ocean. Org Geochem 26:311-320

Keil RG, Fogel ML (2001) Reworking of amino acid in marine sediments: Stable carbon isotopic composition of amino acids in sediments along the washington coast. Limnol Oceanogr 46:14-23

Keil RG, Tsamakis E, Giddings JC, Hedges JI (1998) Biochemical distributions (amino acids, neutral sugars, and lignin phenols) among size-classes of modern marine sediments from the washington coast. Geochim Cosmochim Acta 62:1347-1364 
Khare BN, Sagan C, Ogino H, Nagy B, Er C, Schram KH, Arakawa ET (1986) Amino-acids derived from Titan tholins. Icarus 68:176-184

Kielland K (1995) Landscape patterns of free amino acids in arctic tundra soils. Biogeochemistry $31: 85-98$

Kvenvolden KA, Lawless J, Pering K, Peterson E, Flores J, Ponnamperuma C, Kaplan IR, Moore C (1970) Evidence for extraterrestrial amino-acids and hydrocarbons in Murchison meteorite. Nature 228:923-926

Lawless JG, Yuen GU (1979) Quantification of monocarboxylic acids in the Murchison carbonaceous meteorite. Nature 282:396-398

Lenski RE, Ofria C, Collier TC, Adami C (1999) Genome complexity, robustness and genetic interactions in digital organisms. Nature 400:661-664

Lenski RE, Ofria C, Pennock RT, Adami C (2003) The evolutionary origin of complex features. Nature 423:139-144

Lerner NR, Peterson E, Chang S (1993) The Strecker synthesis as a source of amino-acids in carbonaceous chondrites: Deuterium retention during synthesis. Geochim Cosmochim Acta 57:4713-4723

Lovelock JE (1965) A physical basis for life detection experiments. Nature 207:568-570

Maynard Smith JM (1992) Evolutionary biology - byte-sized evolution. Nature 355:772-773

McCollom TM, Ritter G, Simoneit BRT (1999) Lipid synthesis under hydrothermal conditions by fischer-tropsh-type reactions. Origins Life Evol Biosph 29:153-166

McDonald GD, Khare BN, Thompson WR, Sagan C (1991) CH4/NH3/H2O spark tholin chemical-analysis and interaction with Jovian aqueous clouds. Icarus 94:354-367

McDonald GD, Thompson WR, Heinrich M, Khare BN, Sagan C (1994) Chemical investigation of Titan and Triton tholins. Icarus 108:137-145 
McKay CP (2002) Planetary protection for a europa surface sample return: The ice clipper mission. Advances in Space Research 30:1601-1605

McKay CP (2004) What is life - and how do we search for it in other worlds? PLOS Biology $2: 1260-1263$

Miller SL (1953) A production of amino acids under possible primitive Earth conditions. Science 117:528-529

Miller SL (1955) Production of some organic compounds under possible primitive Earth conditions. J Am Chem Soc 77:2351-2361

Miller SL, Urey HC (1959) Organic compound synthesis on the primitive Earth. Science $130: 245-251$

Munoz-Caro GM, Meierhenrich UJ, Schutte WA, Barbier B, Segovia AA, Rosenbauer H, Thiemann WHP, Brack A, Greenberg JM (2002) Amino acids from ultraviolet irradiation of interstellar ice analogues. Nature 416:403-406

Nagy B, Bitz SMC (1963) Long-chain fatty acids from the orgueil meteorite. Arch Biochem Biophys 101:240

Naraoka H, Shimoyama A, Harada K (1996) Molecular distribution of monocarboxylic acids in asuka carbonaceous chondrites from antarctica. Origins Life Evol Biosph 29:187-201

Ofria C, Adami C, Collier TC (2002) Design of evolvable computer languages. IEEE Trans on Evol Comp 6:420-424

Ofria C, Adami C, Collier TC (2003) Selective pressures on genomes in molecular evolution. J Theor Biol 222:477-483

Ofria C, Wilke CO (2004) Avida: A software platform for research in computation evolutionary biology. Artificial Life 10:191-229

Pace NR (2001) The universal nature of biochemistry. Proc Natl Acad Sci U S A 98:805-808 
Ray TS (1992) An approach to the synthesis of life. In: Langton CG, Farmer JD, Rasmussen S (Eds.) Artificial Life II, Addison-Wesley, Redwood City, pp. 371-408

Ring D, Wolman Y, Miller SL, N F (1972) Prebiotic synthesis of hydrophobic and protein amino-acids. Proc Natl Acad Sci U S A 69:765-768

Rushdi AI, Simoneit BRT (2001) Lipid formation by aqueous fischer-tropsch-type synthesis over a temperature range of 100 to $400^{\circ} \mathrm{C}$. Origins Life Evol Biosph 31:103-118

Schlesinger G, Miller SL (1986) Prebiotic syntheses of pantoic acid and the other components of coenzyme-a. Origins Life Evol Biosph 16:307-307

Schultes EA, Hraber PT, LaBean TH (1997) Global similarities in nucleotide base composition among disparate functional classes of single-stranded RNA imply adaptive evolutionary convergence. RNA 3:792-806

Schultes EA, Hraber PT, LaBean TH (1999) Estimating the contributions of selection and self-organization in RNA secondary structure. J Mol Evol 49:76-83

Shapiro R SMD (2009) The search for alien life in our solar system: Strategies and priorities. Astrobiology 9:335-343

Shimoyama A, Ikeda H, Nomoto S, Harada K (1994) Formation of carboxylic-acids from elemental carbon and water by arc-discharge experiments. Bull Chem Soc Jpn 67:257259

Shimoyama A, Komiya M, Harada K (1991) Low-molecular-weight monocarboxylic acids and gamma-lactones in neogene sediments of the Shinjo basin. Geochem J 25:421-428

Shimoyama A, Naraoka H, Yamamoto H, Harada K (1986) Carboxylic acids in the Yamato791198 carbonaceous chondrites from antarctica. Chem Lett 15:1561-1564

Shimoyama A, Ponnamperuma C, Yanai K (1979) Amino-acids in the Yamato carbonaceous chondrite from Antarctica. Nature 282:394-396

Sugisaki R, Mimura K (1994) Mantle hydrocarbons - abiotic or biotic. Geochim Cosmochim Acta 58:2527-2542 
Summons R, Albrech P, McDonald G, Moldowan J (2008) Molecular biosignatures. Space Science Reviews 135:133-159

Sundh I, Nilsson M, Borga P (1997) Variation in microbial community structure in two boreal peatlands as determined by analysis of phospholipid fatty acid profiles. Appl Environ Microbiol 63:1476-1482

Takano Y, Ohashi A, Kaneko T, Kobayashi K (2004) Abiotic synthesis of high-molecularweight organics from an inorganic gas mixture of carbon monoxide, ammonia, and water by 3 mev proton irradiation. Appl Phys Lett 84:1410-1412

Wakeham SG (1999) Monocarboxylic, dicarboxylic and hydroxy acids released by sequential treatments of suspended particles and sediments of the black sea. Org Geochem 30:10591074

Wang XS, Poinar HN, Poinar GO, Bada JL (1995) Amino acids in the amber matrix and in entombed insects. In: Anderson, Crelling (Eds.) Amber, Resinite, and Fossil Resins, American Chemical Society, Washington, Vol. 617 of ACS Symposium Series, pp. 255262

White DC, Ringelberg DB, Macnaughton SJ, Alugupalli S, Schram D (1997) Signature lipid biomarker analysis for quantitative assessment in situ of environmental microbial ecology. In: Molecular Markers in Environmental Geochemistry, American Chemical Society, Vol. 671 of ACS Symposium Series, pp. 22-34

Wilke CO, Adami C (2002) The biology of digital organisms. Trends Ecol Evol 17:528-532

Wilke CO, Wang JL, Ofria C, Lenski RE, Adami C (2001) Evolution of digital organisms at high mutation rates leads to survival of the flattest. Nature 412:331-333

Yedid G, Bell G (2001) Microevolution in an electronic microcosm. Am Nat 157:465-487

Yuen GU, Lawless JG, Edelson EH (1981) Quantification of monocarboxylic acids from a spark discharge synthesis. J Mol Evol 17:43-47 
Zelles L, Bai QY (1994) Fatty-acid patterns of phospholipids and lipopolysaccharides in environmental samples. Chemosphere 28:391-411 